\title{
EXTRACCIÓN DE COLÁGENO PROVENIENTE DE RESIDUOS DEL PROCESAMIENTO DE Engraulis ringens "ANCHOVETA"
}

\author{
Extraction of collagen from wastes processing Engraulis ringens "anchovy"
}

\author{
Armando Solari ${ }^{1}$ Javier S. Córdova ${ }^{2}$
}

${ }^{1}$ Dirección de Investigaciones Tecnológicas para la Transformación Pesquera (DGITTP) del Instituto Tecnológico de la Producción, ${ }^{2}$ Universidad Nacional Mayor de San Marcos

\section{RESUMEN}

El objetivo del presente trabajo fue extraer el colágeno de los residuos producidos en el procesamiento de anchoveta (conservas y surimi). Para tal efecto, se solubilizaron las proteínas no colagénicas con una solución de hidróxido de sodio o, $1 \mathrm{~N}$ y se neutralizaron con lavados sucesivos con agua ( $\mathrm{pH}$ cercano a neutro). Luego, los residuos fueron descalcificados con una solución de EDTA o,5 M, desengrasados con butanol al 10\% y finalmente se solubilizaron las proteínas colagénicas con ácido acético o,5 $\mathrm{M}$ y se precipitaron con cloruro de sodio 2,6 M. El colágeno precipitado fue dializado y liofilizado. Se cuantificó el contenido hidroxiprolina (Hip) en los residuos y en el colágeno liofilizado, siendo los valores de 6,5 y 52,9 mg de hidroxiprolina/g de muestra, respectivamente. La solubilidad del colágeno liofilizado disminuyó alrededor de $40 \%$ a una concentración de $12 \%$ de $\mathrm{NaCl}$. El gel de electroforesis mostró una banda intensa de peso molecular aproximado de $110 \mathrm{kDa}$ que correspondería a las cadenas $\alpha_{1}$ y $\alpha_{3}$ de la molécula del colágeno tipo I.

Palabras clave: colágeno, anchoveta, solubilidad proteica, hidroxiprolina.

\section{SUMMARY}

The aim of this study was to extract collagen from the waste produced in the processing of anchovies (canned and surimi). To this end, non-collagenous proteins was solubilized with a solution of $\mathrm{o}, \mathrm{N}$ sodium hydroxide and neutralized with successive washes with water (pH close to neutral). Then, the residues were decalcified with a EDTA o, $5 \mathrm{M}$ solution; degreased with butanol $10 \%$ and finally the collagen protein were solubilized with acetic acid o,5 M and precipitated with sodium chloride 2,6M. Collagen precipitated was dialyzed and lyophilized. The hydroxyproline content (Hip) were quantified in waste and lyophilized collagen, getting the values of 6,5 and 52,9 mg of hydroxyproline/g sample, respectively. The solubility of lyophilized collagen decreased about $40 \%$ at a concentration of $12 \% \mathrm{NaCl}$. Gel electrophoresis showed a strong band of approximately $110 \mathrm{kDa}$ molecular weight that corresponds to the $\alpha_{1}$ and $\alpha_{3}$ chains of collagen type I.

Keywords: Collagen, anchovy, protein solubility, hydroxyproline.

\section{INTRODUCCIÓN}

$\mathrm{E}$ n la industria pesquera, los residuos sólidos, constituyen entre el $50-70 \%$ de la materia prima inicial; dependiendo del proceso productivo consisten en una mezcla de cabezas, pieles, huesos y vísceras, principalmente. Aunque su valor nutricional es importante, se les destina a la elaboración de alimento balanceado o fertilizante, con bajo valor agregado $^{(1)}$.

Por otro lado, estudios recientes que se vienen realizando en países tales como Japón, España, Francia, Tailandia y otros, en los que la actividad pesquera es preponderante en su economía, indican que la contaminación a causa del vertimiento de residuos provenientes de esta industria es alarmante y ocasionan serios problemas ambientales.

Investigaciones relacionadas a la composición y caracterización de los residuos de pescado y su utilización en la industria, han informado sobre la obtención de colágeno, gelatina y otras proteínas de alto valor biológico. Gómez-Guillén ${ }^{(2)}$, menciona que el 30\% de los residuos del procesamiento pesquero está conformado por piel y huesos, en los cuales el contenido de colágeno es elevado y cuyo aprovechamiento puede beneficiar a alimentos, cosméticos y elaboración de materiales biomédicos ${ }^{(3)}$.

En el Perú, donde la actividad pesquera representa una fuente importante de divisas, estos problemas de contaminación por residuos constituyen temas de desarrollo que hacen necesarios los estudios de aprovechamiento integral.

En este marco de ideas el presente estudio tiene como objetivo la obtención de colágeno y gelatina a partir de residuos del procesamiento de anchoveta (figura 1).

\section{MATERIAL Y MÉTODOS}

\section{Obtención de residuos}

Se utilizaron los residuos (espinas, espinazos y escamas) de la línea de procesamiento de conservas de filete y surimi de anchoveta, procedente del Callao (figura 2).

Los restos de carne residual adherida a los descartes fueron removidos con agua y con la ayuda de un cepillo de cerdas finas, posteriormente estas muestras fueron cortadas en pedazos de 1-1,5 cm de longitud, empacadas en bolsas de polietileno y congeladas a $-25^{\circ} \mathrm{C}$ hasta su uso.

\section{Composición química proximal y contenido de} hidroxiprolina (Hip)

La determinación de humedad, grasa, cenizas, y proteína (utilizando como factor 5,95 ) en los residuos y el colágeno obtenido se realizó de acuerdo a lo recomendado por FAO ${ }^{(4)}$.

La cuantificación del colágeno fue realizada en base a la determinación de hidroxiprolina (Hip) siguiendo la 
técnica colorimétrica desarrollada por Kolar et al ${ }^{(5)}$.

\section{Extracción de colágeno}

Fue realizada de acuerdo al método propuesto por Nagai y Yamashita ${ }^{(6)}$. Los descartes fueron tratados con hidróxido de sodio o,1N durante 6 horas para la remoción de las proteínas no colagénicas y después lavados con agua corriente para eliminar la soda y alcanzar $\mathrm{pH}$ cercano a la neutralidad. Luego se descalcificó y desengrasó la muestra con EDTA o,5 M y butanol al 10\%, respectivamente.

Para la extracción del colágeno se utilizó solución de ácido acético $0,5 \mathrm{M}$ durante 2 días y se precipitó con $\mathrm{NaCl}$ $2,6 \mathrm{M}$. El precipitado fue llevado a $\mathrm{pH}$ neutro, dializado y liofilizado.

Todos los procedimientos de preparación se realizaron a $4^{\circ} \mathrm{C}$ y los reactivos utilizados en todos los análisis fueron de grado QP.

\section{Solubilidad del colágeno}

La solubilidad del colágeno fue evaluada de acuerdo al método propuesto por Montero et al ${ }^{(7)}$. El colágeno fue disuelto en ácido acético $0,5 \mathrm{M}$ y agitado hasta que fue completamente solubilizado a $4^{\circ} \mathrm{C}$ y obtener una concentración de $6 \mathrm{mg} / \mathrm{mL}$. Cinco mililitros de la solución de colágeno fueron mezclados con $5 \mathrm{~mL}$ de $\mathrm{NaCl}$ en ácido acético $0,5 \mathrm{M}$ en varias concentraciones porcentuales en peso/volumen (o, 2, 4, 6, 8, 10 y 12\%). Las mezclas fueron agitadas continuamente a $4^{\circ} \mathrm{C}$ durante 30 minutos, y centrifugadas a $10000 \mathrm{~g} \mathrm{a} 4^{\circ} \mathrm{C}$ durante 30 minutos. La proteína del sobrenadante fue cuantificada por el método de Lowry et al ${ }^{(8)}$, usando albúmina sérica bovina como estándar. Los resultados fueron expresados como solubilidad porcentual.

\section{Gel de electroforesis}

Para la determinación del peso molecular (PM) del colágeno extraído se preparó un gel de poliacrilamida a una concentración de $15 \%$ (proporción metilenbisacrilamida/acrilamida) de acuerdo a la metodología propuesta por Nagai et al ${ }^{(3)}$.

\section{RESULTADOS}

\section{Composición química proximal, contenido de Hip en los residuos y en el colágeno obtenido}

En la tabla 1, se presentan los valores porcentuales de la composición proximal y de hidroxiprolina en los residuos y el colágeno obtenido, donde se observan las variaciones luego de los tratamientos de extracción así como el incremento de proteínas e hidroxiprolina.

\section{Solubilidad del colágeno}

En la figura 4, se observa la disminución porcentual de la solubilidad del colágeno con el incremento de la concentración de sal. También se observa que a concentraciones mayores al $8 \%$ disminuye su solubilidad casi en $40 \%$ del valor porcentual inicial.
Gel de electroforesis obtenido a partir del colágeno extraído de los residuos de anchoveta

En la figura 5, se puede observar el peso molecular aproximado del colágeno extraído a partir de residuos de pescado, que correspondería a las cadenas $\alpha_{1}$ y $\alpha_{3}$ de la molécula colagénica, siendo su PM aproximado de $110 \mathrm{kDa}$.

\section{DISCUSIÓN}

De la tabla 1 se observa que, en los residuos hay un elevado contenido de humedad $(62,8 \%)$ y bajo valor de proteínas $(10,8 \%)$. El alto contenido de cenizas puede ser explicado por la naturaleza del hueso y su composición, en gran parte debido al calcio que le suministra dureza. Por otro lado, el contenido de grasa fue $7 \%$, valor que puede parecer elevado, sin embargo, considerando un estudio realizado por Kittiphattanabawon et al (1), en el cual hallaron contenidos de grasa de $8,8 \%$ en el hueso de Priacanthus tayenus (inclusive mayor que el reportado para la piel de la misma especie) que podrían ser atribuídos a restos cárnicos, así como a sustancias y componentes de la médula ósea.

En relación al colágeno liofilizado extraído (figura 3), el contenido graso y de ceniza fueron comparativamente menores a los anteriores, indicando la eficacia de la remoción de la materia inorgánica y la grasa durante el proceso de extracción, según lo recomendado por Nagai y Suzuki ${ }^{(3)}$. Finalmente, el contenido de humedad del colágeno extraído de los descartes es menor y el contenido de proteínas fue de $87,6 \%$.

El valor de Hip en el colágeno extraído $(52,9 \mathrm{mg} / \mathrm{g})$ se incrementó casi en 10 veces respecto a su valor inicial en la materia prima, que fue de $6,5 \mathrm{mg} / \mathrm{g}$. Estos resultados concuerdan con lo encontrado por Kittiphattanabawon et al (1), quien refiere valores de Hip en los residuos de $5,7 \mathrm{mg} / \mathrm{g}$ y $58,5 \mathrm{mg} / \mathrm{g}$ para el colágeno extraído de la especie Priacanthus tayenus. Ellos mencionan también que el incremento del contenido de hidroxiprolina está generalmente acompañado del incremento del contenido proteico.

Es necesario indicar que el rendimiento fue alrededor de $1 \%$ con respecto al peso inicial de los residuos, concordando con lo reportado con Kittiphattanabawon et $a l^{(1)}$ quien además señala que el rendimiento a partir de piel es notablemente mayor (10,9\%).

De la figura 4, se observa la disminución de la solubilidad del colágeno, lo cual puede ser atribuído a las interacciones hidrofóbicas y agregación de proteínas debido a su competencia por el agua, causando precipitación proteica ${ }^{(9)}$. Kittiphattanabawon et al (1) señalan que el colágeno proveniente de huesos de pescado es más tolerante a mayores concentraciones de $\mathrm{NaCl}$ debido a que posee diferentes propiedades 


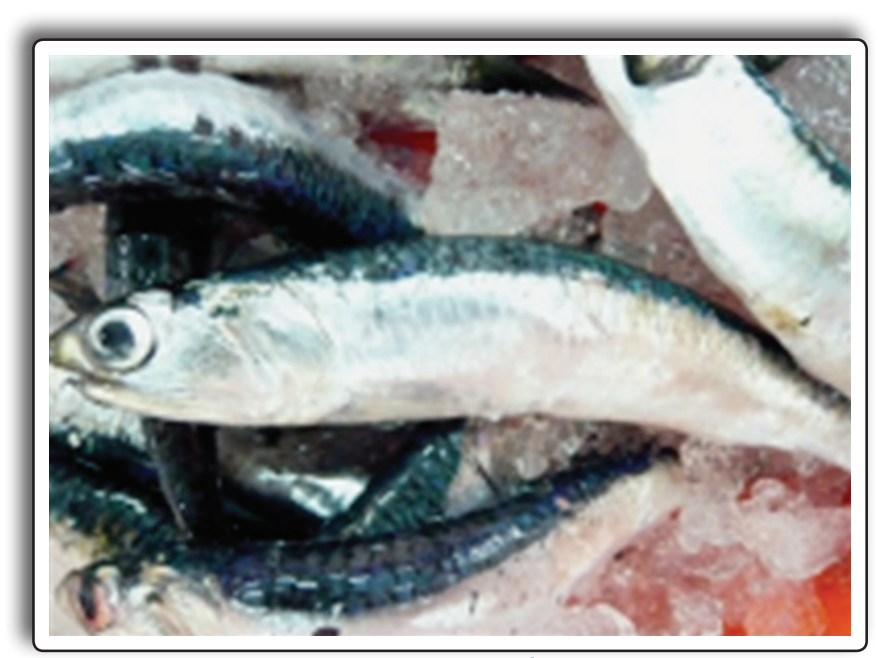

Figura 1. La anchoveta peruana Engraulis ringens.

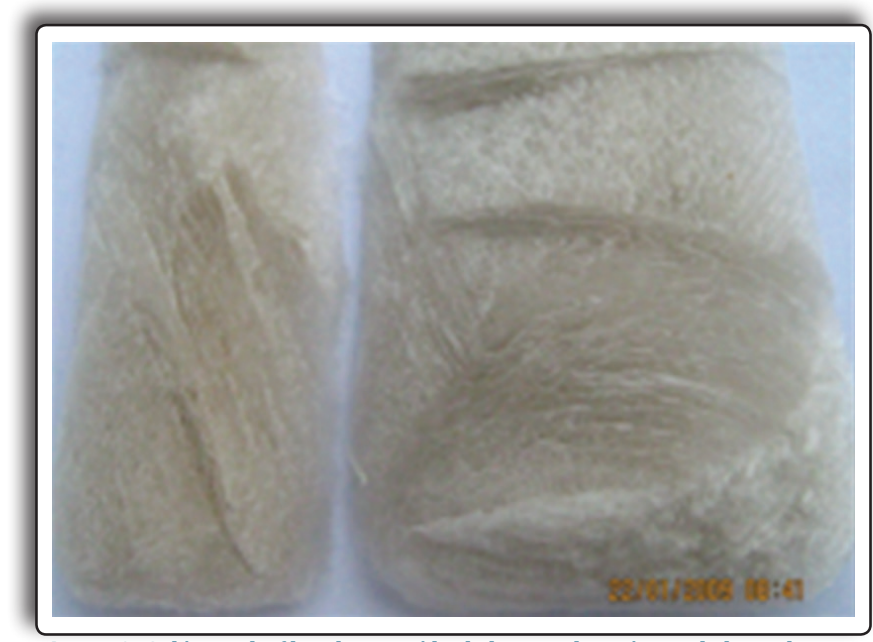

Figura 3. Colágeno liofilizado extraído de los residuos óseos de la anchoveta.

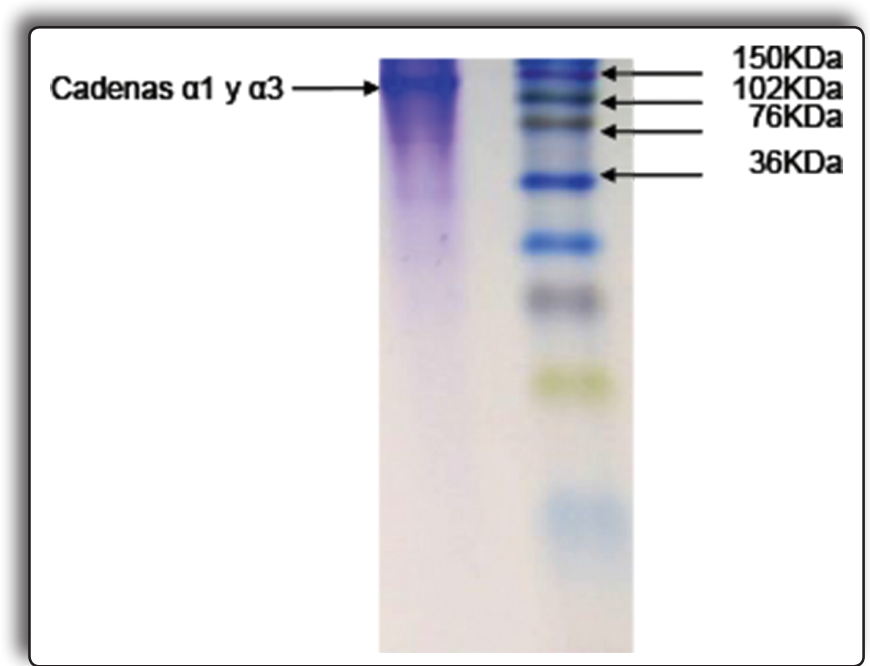

Figura 5. Gel SDS-PAGE al 15\%, identificación del peso molecular del colágeno.

moleculares. Por otro lado, este mismo autor señala que, en medio ácido, la solubilidad del colágeno es

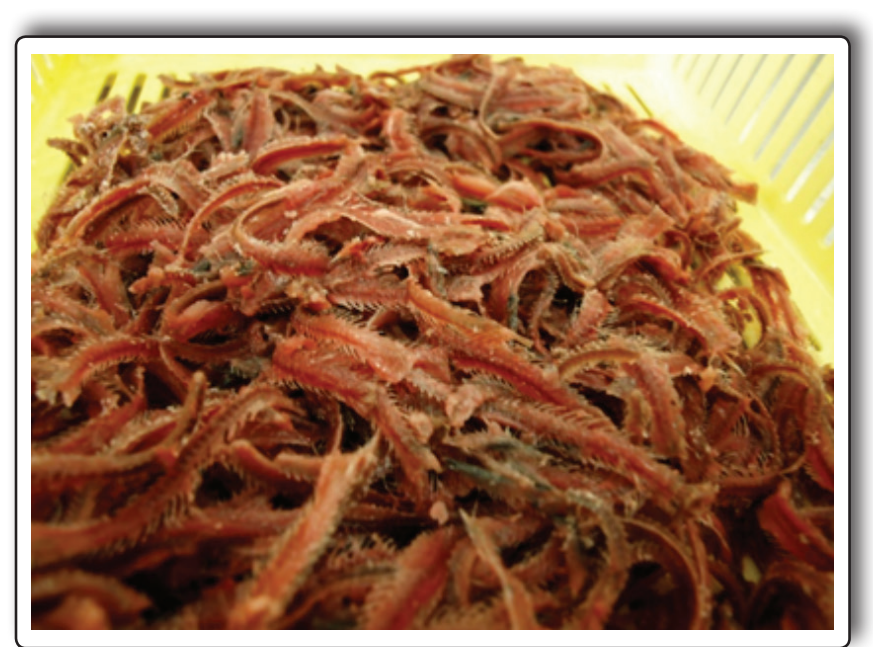

Figura 2. Residuos óseos obtenidos luego del procesamiento de filetes de anchoveta para la elaboración de surimi.

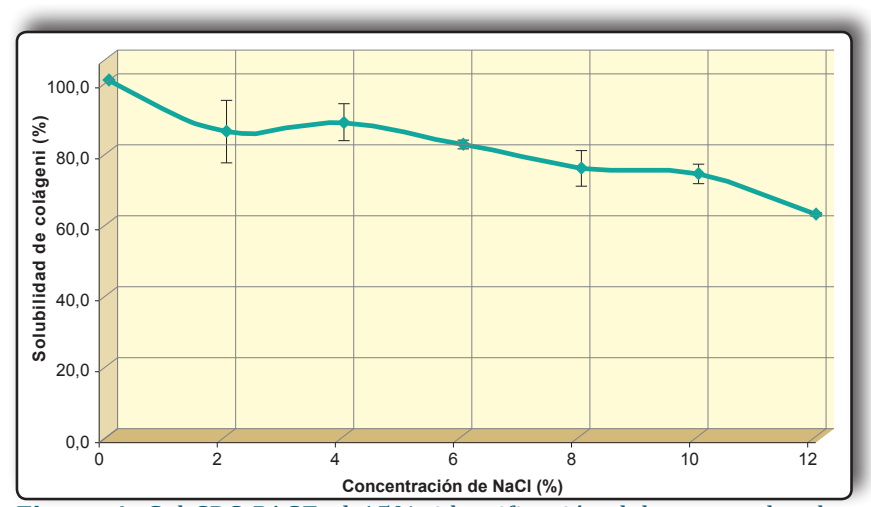

Figura 4. Gel SDS-PAGE al 15\%, identificación del peso molecular del colágeno.

mayor que en rangos de $\mathrm{pH}$ alcalinos y disminuye ante el incremento de sales.

Es necesario mencionar que Montero et al ${ }^{(10)}$ y Ramachandran ${ }^{(11)}$ refieren que el método de extracción de colágeno por liofilización puede causar pérdida de solubilidad de la proteína, debido a la remoción de agua del sistema, afectando a la estructura proteica y ocasionando consecuentemente pérdida de algunas propiedades funcionales.

En la figura 5, se observa la distribución de los pesos moleculares, ya que las cadenas comparten similar estructura molecular y residuos de aminoácidos. Por otro lado, los resultados demuestran que éste material correspondería a colágeno tipo I, tal como lo señala Nagai et al ${ }^{(3)}$, quién identificó los mismos patrones de PM para la especie Japanese sea-bass (Lateolabrax japonicus).

\section{CONCLUSIÓN}

El alto contenido de grasa y cenizas encontrados en los residuos indican que podrían aprovecharse ciertos compuestos de interés comercial. La solubilidad del colágeno obtenido disminuyó con el incremento en la concentración de $\mathrm{NaCl}$ lo cual demostró su funcionalidad. 
La obtención de colágeno a partir de residuos del procesamiento de pescado, es una alternativa que puede ser utilizada para diversificar su uso y generar valor agregado a estas fue ntes. El gel de eletroforesis demostró que el colágeno obtenido correspondió al tipo I.

\section{REFERENCIAS BIBLIOGRÁFICAS}

1. Kittiphattanabawon P, Benjakul S, Visessanguan W, Nagai, Tanaka M. Characterization of acid-soluble collagen from skin and bone of big snapper (Priacanthus tayenus). Food Chemistry. 2005; 89: 363-72.

2. Gómez-Guillén MC, Turnay J, Fernández-Díaz MD, Ulmo N, Lizarbe MA, Montero-García P. Structural and physical properties of gelatin extracted from differents marine species: a comparative study. Food Hidrocolloids. 2002; 16: 25-34.

3. Nagai T \& Suzuki N. Isolation of collagen from fish waste material-skin, bone and fins. Food Chemistry. 2000; 68(15): 277-81.

4. FAO. Food and Nutrition Paper 14/7. Manuals of food Quality Control. Food Analysis: General technics, Aditives, Contaminants and Composition. Prepared by FAO with support of the Swedish International Development Authority (SIDA). Roma; 1986.

5. Kolar K. Colorimetric determination of hydroxyproline as measure of collagen content in meat and meat products: NMKL collaborative study. J Assoc Off Anal Chem. 1990; 73(1): 54-7.
6. Nagai T \& Yamashita E, Taniguchi K, Kanamori N, Suzuki $\mathrm{N}$. Isolation and characterization of collagen from the outer skin waste material of cuttlefish (Sepia lycidas). Food Chemistry. 2001; 72(4): 425-9.

7. Montero $\mathrm{P}$ y Borderías J. Propiedades funcionales del colágeno de pescado en la industria alimentaria. Inf Tec Inv Pesq. 1989; 151.

8. Lowry O, Rosebrough N, Farr A \& Randall R. Protein measurement with Folin phenol reagent. J Biol Chem. 1951; 193: 256-75.

9. Vodjani F. Solubility. Methods of testing protein functionality. $1^{\text {st }}$ ed. Great Britain: St. Edmundsbury Press; In G. M. Hall; 1996.

10. Montero P, Alvarez C, Martí A, Borderías J. Plaice skin collagen extraction and functional properties. J Food Sci. 1995. 60(1): 1-3.

11. Ramachandran G. Stereochemistry of collagen. J Pept Protein Res. 1988; 31(1): 1-16.

Manuscrito recibido el: 05/05/2015

Aceptado para su publicación el: 02/11/2015

\section{Correspondencia}

Nombre: Ing. Javier Saúl Córdova Ramos

Dirección: Jr. Puno 10021002 - Lima o1 - Perú E-mail: $\quad$ javier.cordova.ramos@hotmail.com 